\title{
Total Focussing Method for Volumetric Imaging in Immersion Non Destructive Evaluation
}

\author{
Andrew Tweedie, \\ Alba Ultrasound Limited, \\ Unit 1, Block 3, Todd Campus, \\ West of Scotland Science Park, \\ Glasgow, UK
}

\author{
Richard L O'Leary, Gerald \\ Harvey and Anthony Gachagan \\ Centre for Ultrasonic Engineering, \\ University of Strathclyde, \\ Glasgow, UK
}

\author{
Caroline Holmes, Paul D Wilcox \\ and Bruce W Drinkwater \\ Department of Mechanical \\ Engineering, \\ University of Bristol, \\ Bristol, UK
}

\begin{abstract}
This paper describes the use of a $550(25 \times 22)$ element $2 \mathrm{MHz} 2 \mathrm{D}$ piezoelectric composite array in immersion mode to image an aluminum test block containing a collection of artificial defects. The defects included a $1 \mathrm{~mm}$ diameter side-drilled hole, a collection of $1 \mathrm{~mm}$ slot defects with varying degrees of skew to the normal and a flat bottomed hole. The data collection was carried out using the full matrix capture; a scanning procedure was developed to allow the operation of the large element count array through a conventional 64-channel phased array controller. A 3D TFM algorithm capable of imaging in a dual media environment was implemented in MATLAB for the offline processing the raw scan data. This algorithm facilitates the creation of 3D images of defects while accounting for refraction effects at material boundaries. In each of the test samples interrogated the defects, and their spatial position, are readily identified using TFM. Defect directional information has been characterized using VTFM for defect exhibiting angles up to and including $45^{\circ}$ of skew.
\end{abstract}

Keywords; piezoelectric composite arrays, signal processing, total foccusing method, non-destructive evaluation

\section{INTRODUCTION}

The prime motivation in all modalities of non-destructive evaluation (NDE) is the sizing and classification of defects in engineering components. How this information is then used to make a decision on the on-going course of action will typically depend upon the criticality of the component and the inherent costs, monetary or otherwise, of failure in the component. In ultrasonic NDE, where the defect is large in relation to the propagating wavelength, a number of established techniques can be applied to characterize defects [1]. The difficulty arises where characterization of the criticality of sub-wavelength defects is required - this presents a major challenge to ultrasonic NDE. An obvious solution may be to increase the frequency of the propagating wavefront in order to improve defect resolution. However, factors such as increasing backscatter or attenuation in the component under test may preclude this as a potential solution.

The application of ultrasonic arrays, coupled with improvements in array signal processing offers a solution for the characterization of defects with sub-wavelength dimensions [ 2 ]. Arrays offer increased flexibility and potentially superior performance, when compared to monolithic probes. The time invariant nature of the majority of ultrasonic NDE problems negates the high imaging frame rates often found in the biomedical arena. Therefore the application of ultrasonic arrays to NDE offers the potential to achieve significant improvements in image quality by maximizing the dataset collected by the array. This can be achieved by implementing a data capture methodology in which each array element will in turn act as a transmitter with the remainder of the elements in reception until the complete set of transmitreceive pairs is obtained. Considering an array comprising $n$ elements, $n^{2}$ datasets will result. In this paper this method of data acquisition will be termed full matrix capture and forms the basis for the foregoing signal processing techniques.

Given the full matrix of data from a test piece it is possible to implement in post-processing any number of array imaging algorithms. In particular the application of the total focusing method (TFM) which allows for focusing of the array at every point in the test-piece in order to form the image and has been shown to outperform all other linear imaging algorithms [3]. Furthermore, TFM can be employed beyond obtaining positional information from a reflector in the test-piece - both scalar and vector functions can be obtained in order to describe a defect's position, orientation and specularity.

To date progress has been primarily focused on processing data from linear, directly coupled arrays. In this paper the TFM is applied to the dual media of immersion scanning problems and to considers a high element count 2-dimensional array and as such offers the potential for increased resolution. The next section details the array configuration - this is followed by a description of the test blocks employed to demonstrate the technique. Finally, the results obtained for both TFM and vector-TFM (VTFM) are described.

\section{2D Piezoelectric COMPOSITE ARRAY}

A $550(25 \times 22)$ element $2 \mathrm{D}$ array was employed to demonstrate the potential for volumetric imaging using TFM. The array comprises a piezoelectric composite substrate with a fundamental thickness mode at $2 \mathrm{MHz}$. Figure 1 illustrates the electrical impedance characteristic of a selection of array elements, the response derived using the PZFlex (Weidlinger Associates, Mountain View, CA) finite element code is included for comparison. Electrodes were deposited in sputtered gold with the array elements being defined on one 
face of the composite and a monolithic ground plane on the opposing face - each element has lateral dimension of $0.77 \mathrm{~mm}$ square and a pitch of $2 \mathrm{~mm}$. The piezoelectric composite is mounted onto a light backing. The problem of providing electrical connection to each individual element in high element count arrays is paramount. In the current context, this has been achieved by embedding nickel pins of the correct element pitch into the acoustic backing of the transducer, this was found to be successful with less than $2 \%$ element drop out. Array elements exhibit minimal mechanical cross-talk (-30dB) and $23^{\circ}$ beamwidth $(-10 \mathrm{~dB})$ in water. The completed transducer was marinised in an aluminum housing prior to conducting the testing.

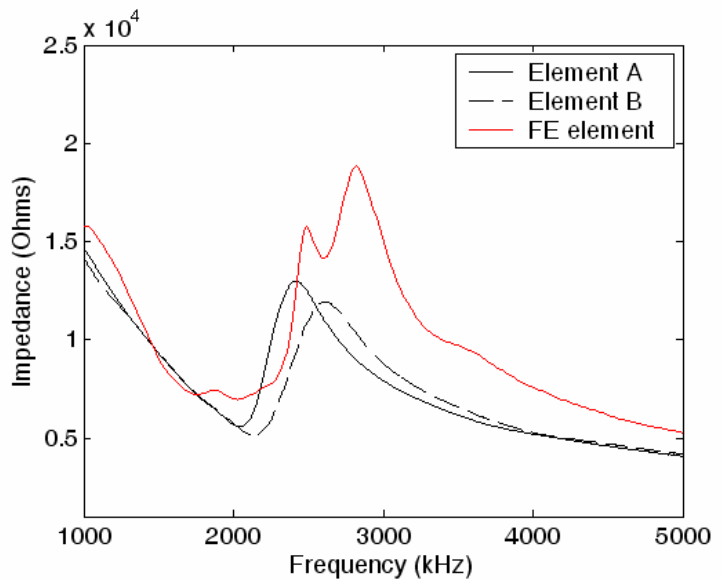

Figure 1 Comparison of measured and PZFlex derived electrical impedance characteristic of a sample of array elements

\section{DATA COLLECTION}

The data were collected using the array described in the previous section and a selection of test pieces with manufactured defects. The test pieces employed in the imaging were both manufactured from aluminum and contained a range of defects. Figure 2 details schematic diagrams of the crosssection of the two test pieces employed in the imaging, for clarity the position of the array in relation to the defect is also shown. In sample 1, shown in Figure 2(a), wire electrical discharge machining (EDM) has been employed to define 6 defects, a $1 \mathrm{~mm}$ diameter through hole and $1 \mathrm{~mm}$ through slots with varying degrees of skew to the normal, $0-60^{\circ}$ in $15^{\circ}$ steps. These defects represent approximately $1 / 3$ of a wavelength at $2 \mathrm{MHz}$, the frequency of operation of the array. Sample 2, shown in Figure 2(b), contains a flat bottomed hole of diameter $1.2 \mathrm{~mm}$, representing approximately $3 / 8$ of a wavelength at $2 \mathrm{MHz}$. During testing, the sample was placed in a water tank and the array suspended such that the front face was positioned $20 \mathrm{~mm}$ from the planar face of the test piece.

The array was operated using a Micropulse MP5PA commercial phased array controller (Peak NDT Ltd, Derby, UK), comprising 64 parallel transmission and reception channels. In order to obtain the full matrix of data from the 550-channel array when only 64 channels are available through the array controller a scanning methodology was implemented. Essentially, each 25-element row of the array was considered as a sub-array, two such sub-arrays were each connected to the array controller and the FMC method carried out on these two sub-arrays. The next pair of 25-element sub-arrays were then attached to the controller and the process repeated until the full matrix of data was collected. The data was stored in the host PC attached to the Micropulse MP5PA for post-processing.

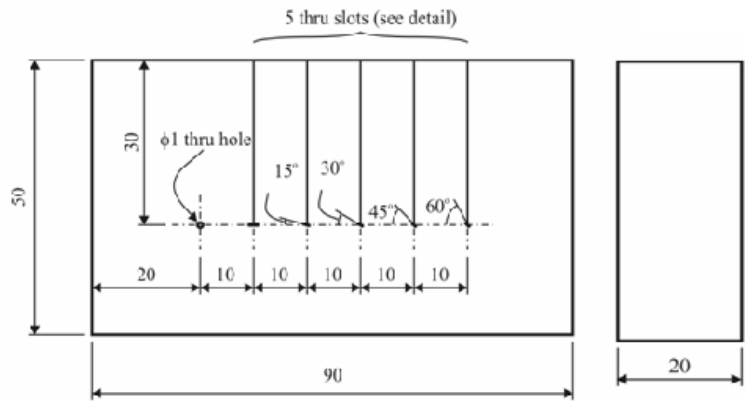

(a)

(Position of the Array)

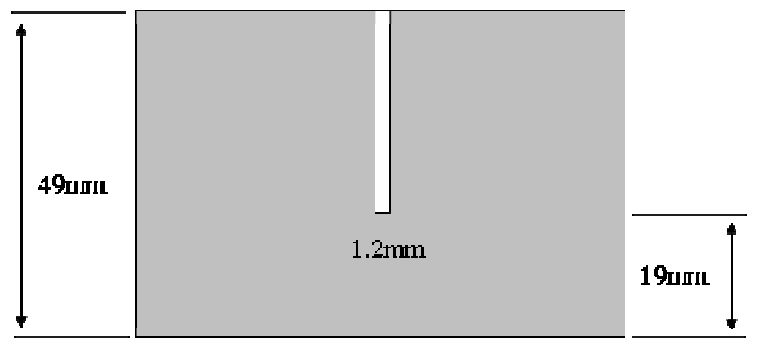

(b)

(Position of the Array)

Figure 2 Schematic diagrams of the test blocks employed in the imaging (a) sample 1 and (b) sample 2

\section{DATA PROCEESING}

The collected data from each of the test pieces was processed in Matlab (Mathworks Ltd, Nantick, MA). The raw data was bandpass filtered using a digital FIR filter with a $-3 \mathrm{~dB}$ bandwidth of $1.4-2.6 \mathrm{MHz}$ and a stopband attenuation of $-40 \mathrm{~dB}$ to remove noise. The filtered data were converted to analytical signals using the Hilbert transform. Since the data were captured using an immersion method, the affect of the path length and transmission of the acoustic energy at liquidsolid interface [4] was accounted for in order to facilitate the TFM.

The results of the TFM for samples 1 and 2 are shown in Figure 3 and Figure 4, respectively. In Figure 3(a), a crosssectional color plot of sample 1 through the vertical plane is shown. The front face reflection and echoes from the back wall of the sample and the mounting plate are also visible. Importantly, the 6 defects are all clearly visible in the plot. Figure 3(b), the defects are shown in more detail by plotting a horizontal cross-section of the TFM data volume. Finally, Figure 3(c) shows the defects in 3D by plotting an iso-surface of the data volume, the position of the array relative to the defect is also shown in this figure. 


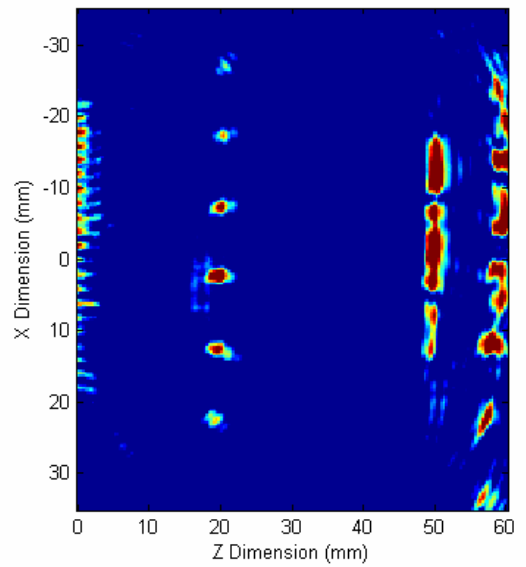

(a)

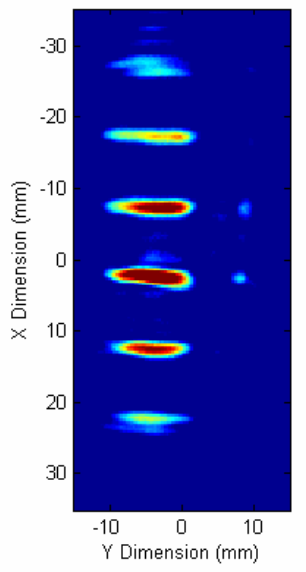

(b)

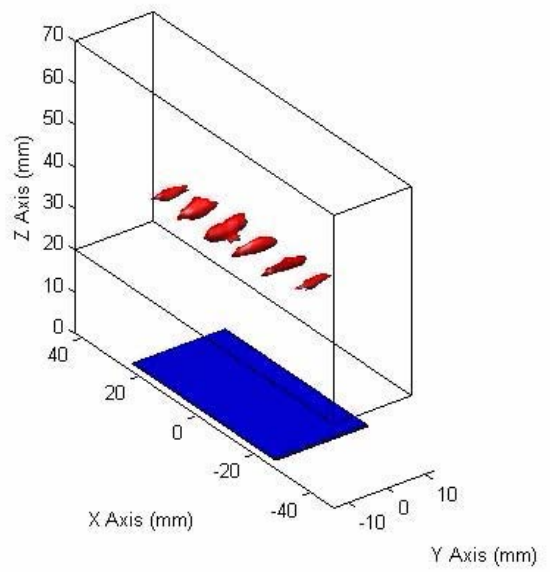

(c)

Figure 3 Imaging results employing TFM on sample 1 (a) side view, (b) plan view and (c) 3D iso-surface with the defects indicated in red and the array position indicated in blue

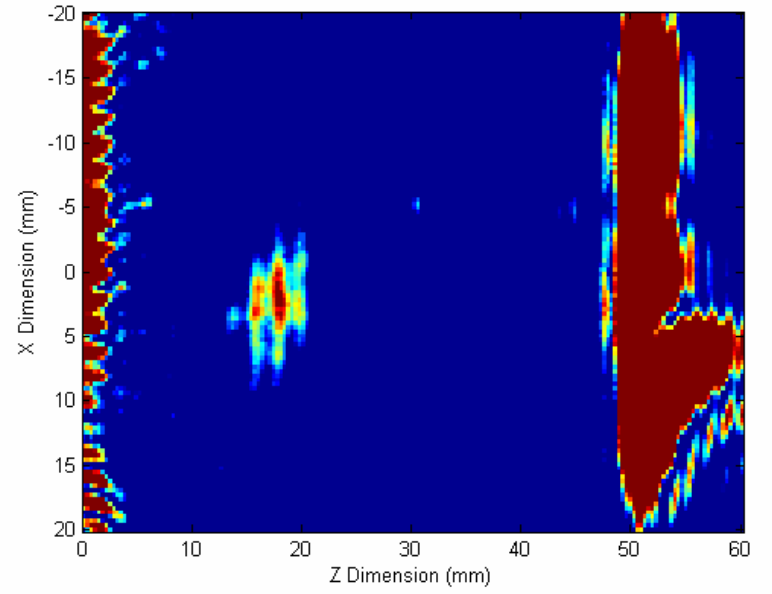

(a)

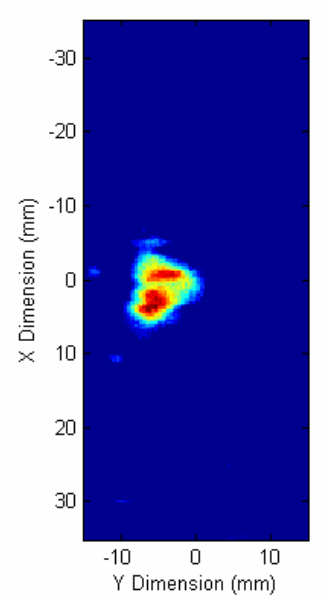

(b)

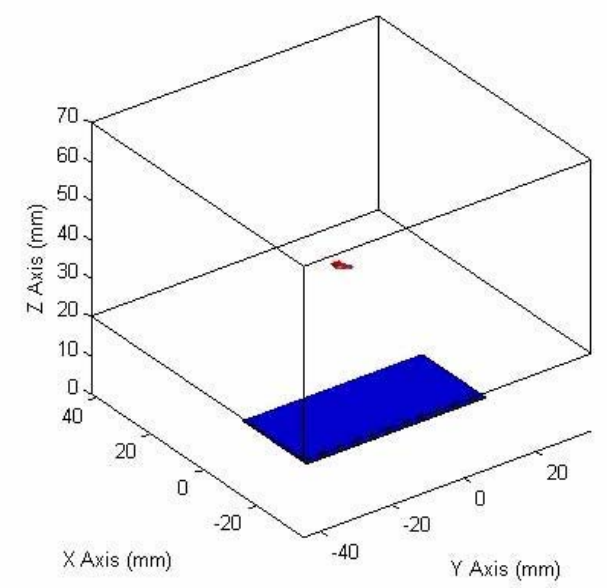

(c)

Figure 4 Imaging result employing TFM on sample 2 (a) side view, (b) plan view and (c) 3D iso-surface with the defects indicated in red and the array position indicated in blue 
In each case the defects are readily identified and the positioning of the defects within the test sample has also been correctly categorized. Considering the TFM data for sample 2, the equivalent data plots are shown in Figure 4.As before, vertical and horizontal cross-sectional color plots are shown, together with an iso-surface plot of the 3D data volume. As in the previous sample, the datasets detailed in Figure 4 clearly show the position of the defect, in this case a flat bottomed hole, in sample 2.

\section{VECTOR TFM}

VTFM allows the directional response of a reflector in a specimen to be determined [2]. The vector field of the specimen is calculated by considering the response of a defect to the subset of array elements. The selection of the subsets of elements may well depend upon the operation of the array - the method can accommodate both static and scanned arrays. In the case of a scanned array the subset of elements could correspond to the entire array at different physical locations. In the current context, the array is static and the subset is selected as a group of contiguous elements in the array. Figure 5 details the results of the VTFM method applied to sample 1. For reasons of brevity only a selection of results are shown, the responses from the 30 and $45^{\circ}$ defects being shown.
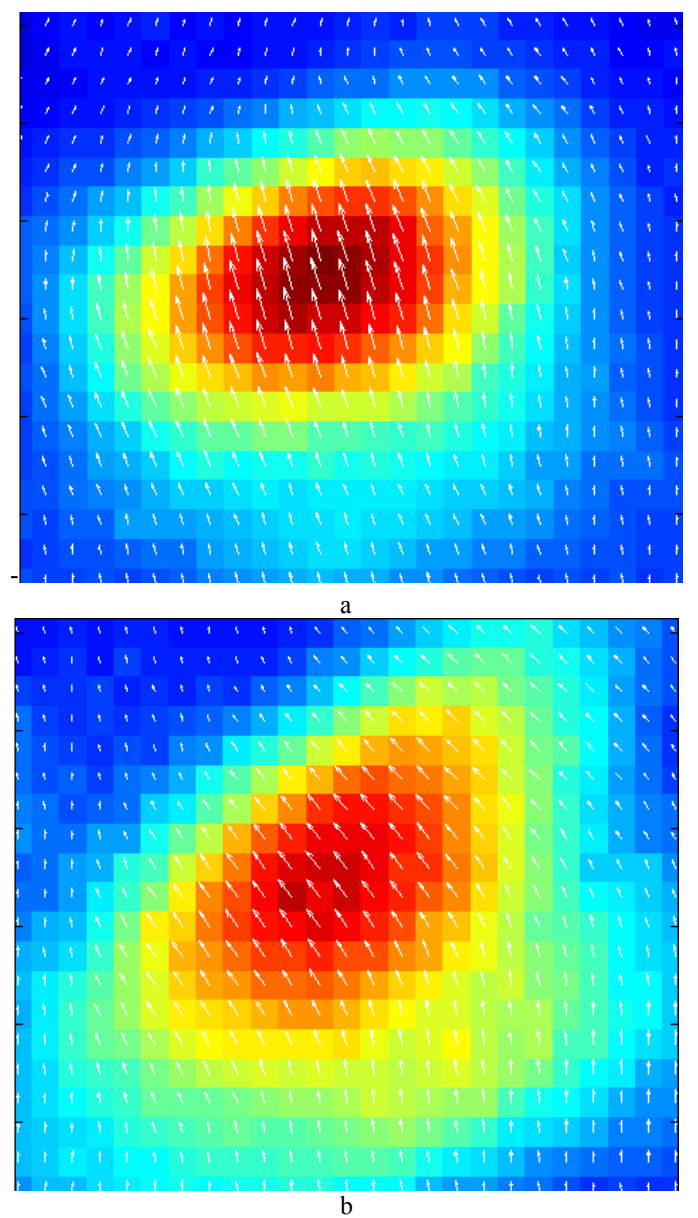

Figure 5 Results of VTFM of sample 1 for (a) $30^{\circ}$ slot and (b) $45^{\circ}$ slot
For reasons of brevity only a selection of results are shown, with Figure 5 detailing the responses from the 30 and $45^{\circ}$ defects. In each of the plots shown in Figure 5 the arrows indicate the direction of the dominant reflection vector overlaid onto the echo response of the defect in question. Importantly, when examining the plots shown in Figure 5 it is clear that only the defect area are contributing to the vector response. The directionality of the $30^{\circ}$ and $45^{\circ}$ defects illustrated in Figure 5 have been shown to be determined using the VTFM methodology. Indeed, the directionality of all of the defects up to the $45^{\circ}$ angle was characterized well using the methodology. However, in the case of the $60^{\circ}$ defect, not shown, the VTFM did not perform as expected; the method determined an angle of $\sim 40^{\circ}$ for this defect. A consequence of the immersion scanning scenario causing a very rapid evanescence of the acoustic energy as the incidence angle at the water-aluminum boundary is increased [4].

\section{CONCLUDING REMARKS}

This paper has described the use of the TFM and VTFM algorithms for the application of volumetric imaging of engineering components using immersion scanning. In the case of the TFM, samples containing a number of manufactured defects have been analyzed. In each case the largest defect dimension was less that half of a wavelength at the frequency of operation of the imaging array. Both 2- and 3-dimensional plots of the defects have been produced illustrating the ability of the technique to identify such sub-wavelength defects. In each case the spatial position of each of the defect has also been correctly resolved.

VTFM has been applied to one of the datasets in order to determine the defect directionality. It has been shown that only the defect areas are contributing to the vector response. It was found that the immersion methodology was capable of characterizing defect direction response up to and including the $45^{\circ}$ defect.

\section{REFERENCES}

[1] F. Chang and G. Andrew, "Ultrasonic pulse-echo contact techniques," in Non-destructive Testing Handbook, Volume 7: Ultrasonic Testing. 2nd ed. A. S. Birks, R. E. Green, and P.McIntire, Eds. Columbus, OH: American Society for Non-destructive Testing, 1991, pp. 193-217.

[2] P.D. Wilcox, C. Holmes and B.W. Drinkwater 'Advanced Reflector Characterization with Ultrasonic Phased Arrays in NDE Applications' IEEE Trans UFFC Vol. 54, No. 8, pp 1541-1550, (2007)

[3] C. Holmes, B. W. Drinkwater, and P. D. Wilcox, "Postprocessing of the full matrix of ultrasonic transmitreceive array data for non-destructive evaluation," Nondestr. Test Eval. Int., vol. 38, no. 8, pp. 701-711, (2005).

[4] L.M. Brekhovskikh, 'Waves in Layered Media' Academic Press, London, (1960) 